ATUALIZAÇÃO

\title{
MECANISMOS IMUNOLÓGICOS
}

NA RESPOSTA CELULAR E HUMORAL

NA LEISHMANIOSE TEGUMENTAR AMERICANA

Luiza de Campos Reis, Maria Edileuza Felinto de Brito, Marina de Assis Souza e Valéria Rêgo Alves Pereira

\section{RESUMO}

\begin{abstract}
A leishmaniose tegumentar americana é uma doença antropozonótica, causada por diferentes espécies de Leishmania, cujas manifestações clínicas variam de lesões cutâneas, que tendem para a cura espontânea, até graves lesões mucocutâneas mutilantes. As infecções por Leishmania podem levar a uma resposta imunológica específica por parte do hospedeiro, caracterizada pelo aumento de células T CD4 ${ }^{+}$e um perfil de citocinas Th1 ou Th2. Infecções por L. major, que é o modelo mais bem estudado, quando ocorrem em camundongos resistentes (como C57BL/6, CBA, $\mathrm{C} 3 \mathrm{H}$ ) estão associadas a uma resposta Th1, enquanto em camundongos susceptíveis (BALB/c) vinculam-se à resposta do tipo $\mathrm{Th} 2$. A resposta imune em humanos não é bem caracterizada como o é em camundongos e envolve o papel das citocinas, das moléculas co-estimulatórias e da saliva do flebótomo. Paralelamente à existência de uma resposta imunológica por parte do hospedeiro, a sobrevivência e a persistência parasitária dependem de estratégias de escape da resposta imune adaptativa.
\end{abstract}

DESCRITORES: Leishmaniose tegumentar americana. Leishmania. Citocinas. Resposta imune.

A leishmaniose tegumentar americana (LTA) é uma doença antropozonótica causada por várias espécies de protozoários do gênero Leishmania, que acomete pele e/ou mucosas e cartilagens e representa um sério problema de saúde pública (WHO, 2003).

No Brasil, apresenta uma incidência de 35.000 casos notificados/ano. As espécies de Leishmania envolvidas na transmissão são representadas por uma espécie do subgênero Leishmania e cinco do subgênero Viannia (Marzochi, 1992; FUNASA, 2002). A transmissão da doença ocorre no ato da hematofagia pelas

1 Centro de Pesquisas Aggeu Magalhães - CPqAM/FIOCRUZ.

Endereço para correspondência: Dra. Valéria Pereira. Centro de Pesquisas Aggeu Magalhães, Av. Moraes Rego, s/n, Cidade Universitária, Cx. Postal 7472, CEP: 50670-420, Recife, Pernambuco, Brasil. Fax: 55 (81) 3453-2449 e 55 (81) 2101-2631. Email: valeria@cpqam.fiocruz.br

Recebido para publicação em 3/6/2005. Revisto em 9/8/2006. Aceito em 14/8/2006. 
fêmeas infectadas de dípteros pertencentes ao gênero Phlebotomus, no Velho Mundo, e ao gênero Lutzomyia, no Novo Mundo (WHO, 2003).

A Leishmania apresenta as formas promastigota e amastigota. As formas promastigotas metacíclicas são flageladas, responsáveis pela transmissão da doença e encontradas no inseto vetor, enquanto as amastigotas são encontradas no sistema fagocítico do hospedeiro vertebrado (Hoare \& Wallace, 1966). As formas clínicas da LTA dependem de fatores do parasito, do vetor e de suas características epidemiológicas, além da constituição genética e imunológica do hospedeiro.

A infecção pode ser assintomática ou apresentar um espectro que varia desde lesões cutâneas localizadas ou disseminadas, forma cutânea-difusa, até graves lesões mucocutâneas (Grimaldi \& Tesh, 1993). As manifestações clínicas estão geralmente associadas a uma espécie de Leishmania, embora algumas espécies possam causar diferentes formas clínicas (Garcia et al., 2005).

São seis as espécies encontradas no Brasil: Leishmania (Viannia) braziliensis, responsável pela leishmaniose cutânea localizada, disseminada e mucocutânea; Leishmania (Viannia) guyanensis, Leishmania (Viannia) lainsoni, Leishmania (Viannia) shawi, Leishmania (Viannia) naiffi, responsáveis pela leishmaniose cutânea localizada e Leishmania (Leishmania) amazonensis, responsável pela leishmaniose cutânea localizada e difusa (Lainson \& Shaw, 1987; Gramiccia \& Gradoni, 2005).

Odiagnóstico é feito pela associação dos aspectos clínicos, epidemiológicos e laboratoriais (Manson-Bahr, 1987; Kar, 1995). Infecções parasitárias podem levar a uma resposta imunológica específica por parte do hospedeiro, caracterizada pelo aumento de células T CD4 ${ }^{+}$e um perfil de citocinas Th1 ou Th2 (Kemp, 1997; Bogdan \& Rollinghoff, 1998). Paralelamente a essa resposta, a sobrevivência da Leishmania depende da evasão do sistema imune do hospedeiro e esse parasito é altamente adaptado ao escape dos efeitos da imunidade humoral e da imunidade celular (Sacks \& Sher, 2002; Zambrano-Villa et al., 2002).

Nessa atualização, abordaremos os mecanismos imunológicos envolvidos na resposta do hospedeiro, nos modelos murino e humano, ante a infecção por LTA, além das estratégias de evasão do parasito à resposta imune do hospedeiro.

\section{ASPECTOS IMUNOLÓGICOS DA LTA EM MODELO MURINO}

A infecção experimental em camundongos tem sido utilizada para examinar aspectos da relação parasito-hospedeiro na leishmaniose, como o controle genético de susceptibilidade e resistência, o papel da resposta imune mediada por células e a interação parasito-macrófago (Handman et al., 1979; Scott \& Farrell, 1998).

A infecção em camundongos com promastigotas de L. major é o melhor modelo no estudo das doenças crônicas infecciosas que envolvem a ativação de células T CD4 ${ }^{+}$(Scharton \& Scott, 1993; Scott \& Farrel, 1998). Os camundongos 
geneticamente susceptíveis desenvolvem lesões cutâneas no sítio de inoculação e falham no desenvolvimento de uma resposta imune ao parasito mediada por células; já os camundongos resistentes parecem curar-se rapidamente, graças a uma forte resposta imune celular, e mostram-se resistentes a novas reinfecções. A resistência é conferida por células Th1, enquanto a susceptibilidade é conferida por células Th2 (Locksley et al., 1987; Awasthi et al., 2004). A dicotomia Th1 X Th2 é observada nesse modelo e está associada à produção de IL-4 em camundongos susceptíveis e de IFN- $\gamma$ em camundongos resistentes, no que concerne à $L$. major (Heinzel et al., 1989).

Muitas linhagens de camundongos resistentes, como C57BL/6, CBA e $\mathrm{C} 3 \mathrm{H}$, desenvolvem uma doença autolimitada quando infectadas com L. major ou L. tropica. Neste caso, apresentam um aumento na produção de IL-12, que ativa células $\mathrm{NK}$, e células $\mathrm{T} \mathrm{CD} 4^{+}$e $\mathrm{CD}^{+}$para produzirem IFN- $\gamma$, necessário para $\mathrm{o}$ desenvolvimento da resposta Th1 (Howard et al., 1980; Rogers et al., 2002).

Linhagens susceptíveis, como BALB/c, apresentam uma doença progressiva e severa, com um aumento na expressão de mRNA para IL-4 e na produção de IL-5, IL-10 e IL-13 (Howard et al., 1980; Scott \& Farrel, 1998; Himmelrich et al., 2000). IL-4 diminui a regulação da expressão da subunidade $\beta$ dos receptores da IL-12 nas células Th1, suprimindo o desenvolvimento de IFN- $\gamma$, o que leva ao desenvolvimento da resposta Th2 (Wang et al., 1994; Carrera et al., 1996).

A IL-10 desempenha um papel fundamental na inibição da ativação macrofágica e contribui para o crescimento do parasito nas lesões, uma vez que camundongos BALB/c IL10-/ mostraram-se capazes de controlar a progressão da doença durante infecção por L. major (Kane \& Mosser, 2001). Tem sido demonstrado que células NK representam uma fonte inicial de IFN- $\gamma$, que é um importante mediador da resistência inata contra o parasito (Becker et al., 2003). Camundongos com imunodeficiêncica combinada severa (SCID), os quais têm perda das funções de células T, mas possuem células NK normais, são capazes de controlar os parasitos de L. major. A neutralização de IFN- $\gamma$ ou depleção dessas células NK, antes da infecção, inibe a habilidade que esses animais têm para controlá-la (Laskay et al., 1993; Korbel et al., 2004). Anticorpos anti-Leishmania têm sido demonstrados in vitro promovendo a lise dos promastigotas na presença do Complemento, o que facilita a fagocitose (Liew, 1990). Outro fator que influencia a resposta do tipo Th1 ou Th2 é a saliva de algumas espécies de flebótomos que contém substâncias que facilitam a infecção (Gillespie et al., 2000). Duas são as moléculas mais investigadas. A primeira é a Maxadilan (MAX), presente nas glândulas salivares de Lutzomyia longipalpis, que inibe as funções das células $\mathrm{T}$ e dos macrófagos em relação à produção de $\mathrm{NO}, \mathrm{H}_{2} \mathrm{O}_{2}$ e à secreção de TNF$\alpha$ e aumenta a secreção de prostaglandina $\mathrm{E}_{2}$. A segunda é a Apyrase, molécula vasodilatadora mais abundante em Phlebotomus papatasi, que inibe as funções das células T e também a produção de citocinas Th1, aumentando a produção de IL-4 e ocasionando uma infecção exacerbada (Mbow et al., 1998; Gillespie et al., 2000). 


\section{RESPOSTA IMUNE HUMANA E SUAS FORMAS CLÍNICAS}

Em humanos, a resposta imune à infecção por Leishmania não é tão bem caracterizada como a resposta em camundongos, em virtude de sua complexidade. Esta resposta envolve o papel das citocinas, das moléculas co-estimulatórias e da saliva do flebótomo (Rogers et al., 2002).

Em todas as formas clínicas da LTA, a resposta imune é dependente de células $\mathrm{T}$ e, de maneira geral, aceita-se que a diferença entre resistência e susceptibilidade à infecção por Leishmania está relacionada com o nível de expansão de células Th1 e Th2 (Pirmez et al., 1993; Bacellar et al., 2002). Pacientes que contraem a forma cutânea localizada desenvolvem uma resposta do tipo Th1 no nível das lesões. As lesões mucocutâneas, que constituem uma forma crônica associada à destruição de mucosas, caracterizam-se por uma mistura de resposta dos tipos Th1 e Th2, enquanto pacientes com a forma difusa exibem uma resposta quase exclusivamente do tipo Th2 (Cáceres-Dittmar et al., 1993; Pirmez et al., 1993).

Na leishmaniose humana, o papel da resposta imune celular do tipo Th1 tem sido associado ao controle da infecção. O evento crucial para indução da resposta imune curativa contra a Leishmania éa eficiente ativação de células capazes de produzir citocinas protetoras que levem à ativação de macrófagos, via IFN- $\gamma$, resultando na síntese de intermediários de nitrogênio e oxigênio reativo e, conseqüentemente, na morte dos parasitos intracelulares (Salaizo-Suazo et al., 1999).

Há um consenso geral de que as células $\mathrm{T}$ e a imunidade mediada por células contribuem para a patogênese das diferentes formas de LTA. Embora estudos avaliem a resposta humoral na LTA, ainda não está completamente esclarecido o papel de anticorpos específicos na imunidade contra Leishmania (Trujillo et al., 1999; Souza et al., 2005). Nas leishmanioses cutânea e mucocutânea, a imunidade celular e a predominância de isotipos IgG1, IgG2 e IgG3 têm sido associadas à resposta do tipo Th1; já o perfil Th2 tem sido relacionado com a leishmaniose cutânea difusa, com presença de IgG4 (Rodriguez et al., 1996; Souza et al., 2005). Pacientes com um maior tempo de evolução da doença apresentam altos níveis de $\operatorname{IgE}$ e em pacientes com a forma mucocutânea, os níveis de IgA se mostram aumentados (O'Neil et al., 1993). Correlação positiva entre os níveis de IgE com o tamanho da intradermorreação e uma correlação inversa entre os níveis de IgE com o número de lesões foram observadas por Sousa-Atta et al. (2002).

A intensidade da resposta humoral parece estar relacionada com a carga parasitária e com a cronicidade da infecção. Podem ser observados altos títulos de anticorpos em todas as manifestações clínicas da LTA (Trujillo et al., 1999).

$\mathrm{Na}$ forma cutânea localizada, as lesões são geralmente ulceradas e apresentam poucos parasitos com tendência para a cura espontânea ou uma boa resposta ao tratamento (da-Cruz et al., 2002). Há uma forte resposta de células T, com citocinas do tipo Th1, como IFN- $\gamma$ e IL-12, e uma alta freqüência de células B (Louzir et al., 1998; Vieira et al., 2002). 
A leishmaniose cutânea disseminada é caracterizada por várias lesões acneiformes e papulares, com parasitos raros ou ausentes, é freqüente o envolvimento das mucosas. As respostas imunológicas são bem variadas, tanto há uma forte resposta de células T como uma ausência de imunidade celular (Carvalho et al., 1994).

Indivíduos com leishmaniose cutânea difusa apresentam múltiplos nódulos e falham em produzir uma resposta imune mediada por células durante a infecção, sendo incapazes de controlar a multiplicação parasitária e a progressão da doença em conseqüência da falta da expressão de mRNA para IFN- $\gamma$ e da baixa expressão para IL-2. Apresentam ainda elevados títulos de anticorpos específicos contra Leishmania (Bomfim et al., 1996; Trujillo et al., 1999). Esses pacientes não apresentam cura, são altamente resistentes à quimioterapia e exibem uma resposta do tipo Th2, com elevados níveis de IL-4 (Cáceres-Dittmar et al., 1993; Bomfim et al., 1996).

A leishmaniose mucocutânea (LM) é caracterizada por lesões destrutivas das mucosas, principalmente boca e nariz, não ocorrendo cura espontânea. Apresenta uma elevada resposta por células $\mathrm{T}$ específicas, tanto Th1 como Th2, sendo direcionada para uma resposta do tipo Th1 que é mais forte quando comparada com lesões cutâneas localizadas. A resposta exacerbada do tipo Th1 está associada a uma crônica e severa destruição tecidual, em razão de uma forte resposta inflamatória, e à escassez de parasitos nas lesões (Marsden, 1986; Bacellar et al., 2002; Matos et al., 2005). Altos níveis de citocinas pró-inflamatórias TNF- $\alpha$ e IFN- $\gamma$ são produzidas, além de IL-4. Essa resposta é fracamente regulada por IL-10 e TGF- $\beta$, encontradas em níveis diminuídos, mostrando que uma resposta inadequada do tipo Th1, considerada protetora na maioria das formas dessa doença, pode levar a uma imunopatogênese exacerbada (Bacellar et al., 2002; Amato et al., 2003). A LM está também associada a elevados níveis de anticorpos circulantes, como IgG1 e, principalmente, IgG3 (Rodriguez et al., 1996; Junqueira Pedras et al., 2003). A ativação de células T é também influenciada pela co-estimulação dessas células pelas células que apresentam antígenos. A co-estimulação através da molécula CD28 nas células T resulta na eficiente ativação e proliferação de células $\mathrm{T}$ induzidas pelo antígeno e prolonga a sobrevivência celular e a produção de citocinas (Elloso \& Scott, 1999).

Estudos sugerem que a expressão de CD40, CD80 e CD86 tem um impacto significante na resposta imune anti-Leishmania. O bloqueio dessas moléculas promoverá significante inibição na produção de IFN- $\gamma$, IL-5 e IL-12 (Brodskyn et al., 2001). Recentes estudos demonstram que a reposta Th1, formada após infecção por $L$. braziliensis, é acompanhada pela resposta de células T produtoras de IL-10 (Antonelli et al., 2004). Citocinas como IFN- $\gamma$, TNF- $\alpha$ e IL-12 podem ser tóxicas quando produzidas em quantidades elevadas e a IL-10 bloqueia a ativação de células Th1 e, conseqüentemente, previne a superprodução dessas citocinas, evitando dano tecidual (Ribeiro-de-Jesus et al., 1998). A IL-10 também inibe a ativação macrofágica, como a expressão de moléculas co-estimulatórias como B7.1 (CD80) e B7.2 (CD86) e moléculas de adesão intercelular, diminuindo a habilidade dessas células em destruir a Leishmania (Ding et al., 1993; Bourreau et al., 2001). 
Da mesma forma como foi demonstrado em modelo murino, as células NK também são decisivas na resposta de cura e eliminação de parasitos na leishmaniose humana (Maasho et al., 1998). Dois fatos têm sido demonstrados: promastigotas de $L$. donovani e $L$. aethiopica ativam células NK humanas a secretarem IFN- $\gamma$ na ausência de outras células que apresentam antígeno; a estimulação direta de TLR (receptor toll-like)-tipo 2 de células NK pelo lipofosfoglicano (LPG) de L. major leva a uma expressão elevada desse receptor e aumenta a produção de IFN- $\gamma$ e TNF- $\alpha$, sugerindo a existência de uma rota adicional independente de células acessórias na ativação de células NK na leishmaniose (Becker et al., 2003; Nylen et al., 2003; Korbel et al., 2004).

A resposta imunológica humana também é influenciada pelas moléculas contidas na saliva dos flebótomos, como proteínas, enzimas e prostaglandinas. Além de serem anticoagulantes e vasodilatadores, atuam na supressão da resposta inflamatória e na modulação de citocinas (Gillespie et al., 2000). Segundo Coler \& Reed (2005), a quimioterapia, associada à resistência a drogas e à toxicidade, enfatiza a necessidade de uma vacina eficaz contra a leishmaniose que induza uma correta resposta imune, como, por exemplo, uma resposta Th1. Os primeiros estudos foram realizados nos países asiáticos, onde foram inoculadas cepas virulentas vivas de Leishmania na população, prática conhecida como leishmanização, cujo objetivo seria a imunidade duradoura à reinfecção após a cura da lesão, o que tem sido eficaz na leishmaniose cutânea no Velho Mundo (Khamesipour et al., 2005).

Desde a década de 1980, têm sido realizados estudos visando obter uma conduta terapêutica alternativa para LTA (Mayrink et al. 1979, 1992, 2006). Machado-Pinto et al. (2002) associaram o antimônio com uma vacina de promastigotas mortas de L. amazonensis e observaram que essa imunoquimioterapia foi altamente eficaz no tratamento da LTA. Vacinas utilizando preparações antigênicas de várias espécies de Leishmania, com ou sem BCG como adjuvante, têm sido testadas tanto para a forma cutânea como para a visceral (Coler \& Reed, 2005). Moléculas imunogênicas presentes na saliva de flebótomos também podem atuar como adjuvantes no desenvolvimento de vacinas, visto que, em modelo experimental, a pré-exposição à saliva protege contra uma infecção exacerbada causada por $L$. major e L. amazonensis, sugerindo que uma vacina baseada na saliva de flebótomos pode ser efetiva (Gillespie et al., 2000; Kamhawi, 2000).

Progressos também ocorreram nos estudos de novos antígenos candidatos a vacinas de segunda geração contra leishmaniose. Para uma vacina ideal, esses antígenos, como LmSTI1, TSAe LeIF, preferencialmente devem estar conservados nas diferentes espécies de Leishmania expressas abundantemente na superfície do parasito (Coler \& Reed, 2005). Dados experimentais têm demonstrado que a combinação entre células $\mathrm{T}$ de memória central e de memória efetora é mais efetiva no combate às infecções (Zaph et al., 2004). Ainda permanecem alguns questionamentos em relação a essas células e maiores investigações são necessárias, de modo que contribuam para o desenvolvimento de vacinas contra Leishmania (Gollob et al., 2005). 
Uma característica comum em infecções por Leishmania, após cura clínica da doença, é a persistência parasitária. Vários mecanismos imunológicos, como modulação das atividades microbicidas do hospedeiro, síntese de citocinas inibitórias, falha na ativação de células $\mathrm{T}$ ou isolamento do parasito dentro de células que não produzem uma resposta imune, promovem a persistência do parasito. Esse fato talvez possa ser explicado como uma garantia da transmissão e, conseqüentemente, da manutenção do ciclo do parasito (Aebischer, 1994; Mendonça et al., 2004). A demonstração dessa persistência em indivíduos curados clinicamente levanta várias questões a respeito da evolução clínica, da epidemiologia e das estratégias para o controle da leishmaniose (Mendonça et al., 2004).

\section{ESTRATÉGIAS DE EVASÃO DO PARASITO}

As infecções por Leishmania são caracterizadas pela habilidade que têm estes parasitos para escapar da destruição extracelular e penetrar em células fagocíticas, onde vão resistir ao seu poder antimicrobiano, persistindo mesmo na presença de resposta imune celular do hospedeiro (Bogdan et al., 1996; Cunningham, 2002; Rogers et al., 2002).

Amanifestação da doença requer mecanismos que permitam a replicação do parasito no hospedeiro e sua resistência, pelo menos inicialmente, às defesas imunes inatas e adquiridas. O primeiro mecanismo de escape dos promastigotas é evitar a lise direta através do sistema Complemento, tendo a participação de glicoproteínas e açúcares da membrana do parasito, do LPG e da metaloproteinase gp63. Estes atuam por fosforilação, inativando componentes do sistema Complemento como o C3, C5 e C9, com a subseqüente inibição das vias clássicas e alternativas do Complemento. Também impedem o acesso do complexo de ataque à membrana (C5b-C9). A gp63 protege o parasito através da acelerada conversão proteolítica na superfície do parasito do C3b para C3bi que funciona como uma opsonina, facilitando a ligação com receptores do Complemento tipo 3 (CR3) nos macrófagos (Bogdan et al., 1996; Bogdan \& Rollinghoff, 1998). Ao escaparem do meio extracelular, os parasitos irão penetrar nas células fagocíticas através da interação com os receptores dos macrófagos e com as moléculas da superfície do parasito (Sacks \& Sher, 2002). Essa ligação pode ser direta, via parasito-macrófago, ou indireta, através de moléculas do soro associadas com moléculas do parasito e com os macrófagos. Geralmente os promastigotas são opsonizados pelos $\mathrm{C} 3 \mathrm{~b}$ e $\mathrm{C} 3 \mathrm{bi}$, que se fixam respectivamente nos CR1 e CR3 do macrófago. Outra molécula de superfície do parasito, a GIPL (glicoinositolfosfolipídeo), ao que tudo indica tem um importante papel na ligação do parasito ao macrófago, uma vez que, em estudos anteriores, anticorpos contra essa molécula bloquearam a internalização do parasito (Bogdan \& Rollinghoff, 1998). Portanto, para o estabelecimento da infecção, a Leishmania precisa sobreviver ao processo de fagocitose que envolve a invasão em células-alvo seguras, a inibição da formação do fagolisossomo e a remoção dos radicais hidroxilas e ânions superóxidos 
através do LPG, a inibição da degradação das enzimas do fagolisossomo pela gp63, a transformação em amastigotas que são mais resistentes às enzimas óxido nítrico (NO) e peróxido de hidrogênio $\left(\mathrm{H}_{2} \mathrm{O}_{2}\right)$ e ao $\mathrm{pH}$ ácido do fagolisossomo.

A Leishmania utiliza outros mecanismos que auxiliam nas estratégias de escape da defesa do hospedeiro. A modulação de citocinas pode ocorrer através da inibição/desativação de macrófagos, pela supressão ou perda da indução da ativação de citocinas; a inibição da apresentação de antígeno e da estimulação de células T, pela supressão, internalização e degradação de moléculas MHC II e a alteração da função/diferenciação de células T, através da indução e expansão de células do tipo Th2 (Bogdan \& Rollinghoff, 1998; Cunningham, 2002; Forget, 2004). Os antígenos são processados dentro dos macrófagos e apresentados às células $\mathrm{T} \mathrm{CD}^{+}$que determinarão uma resposta do tipo Th1 ou Th2. O tipo de cepa e a dose inoculada do parasito, o sítio de inoculação, a saliva de algumas espécies de flebótomos, aspectos imunológicos e predisposição genética do hospedeiro podem influenciar nessa resposta (Rogers et al., 2002). Também há evidências de que as formas amastigotas do parasito, opsonizadas por IgG, podem ser fagocitadas via receptor Fc dos macrófagos. Essa ligação do patógeno com receptores dos macrófagos pode alterar o perfil de citocinas secretadas, prevenindo a produção de citocinas proinflamatórias (Pleass \& Woof, 2001).

Kima et al. (2000), utilizando camundongos nocaute para cadeia $\gamma$ do receptor $\mathrm{Fc}$, mostraram que esses animais foram refratários à infecção do parasito do complexo L. mexicana. Além disso, experimentos com camundongos nocaute para a citocina IFN- $\gamma$ indicaram que a ligação de formas amastigotas de Leishmania ao $\mathrm{Fc} \gamma \mathrm{R}$ da superfície de macrófagos, via IgG do hospedeiro, resulta na produção de altos níveis de IL-10 e, conseqüentemente, bloqueia a habilidade dos macrófagos de matar os parasitos (Kane \& Mosser, 2001).

Recentes estudos sugerem que microrganismos patogênicos podem modular ou interferir no reconhecimento de padrões mediados pelos receptores toll-like (TLRs) e podem usar os TLRs como um mecanismo de escape do hospedeiro (Netea et al., 2004). Os TLRs reconhecem padrões moleculares associados a patógenos (PAMPs) e isso induz à produção de sinais responsáveis pela ativação de genes importantes para uma defesa imune do hospedeiro, especialmente de citocinas pró-inflamatórias. A ausência de sinalização intracelular sobre a ligação de PAMPs com TLRs resulta no aumento da susceptibilidade a parasitos, como L. major (de Veer et al., 2003). Embora outros TLRs possam estar envolvidos, camundongos nocautes em TLR-tipo $4(\mathrm{C} 3 \mathrm{H} / \mathrm{HeJ})$ são capazes de iniciar uma resposta imune inata contra antígenos de L. braziliensis (Borges et al., 2001).

Hawlisch et al. (2005) verificaram o efeito supressor do Complemento C5a na polarização para Th1, no qual foi evidenciada, pela resistência do camundongo $\mathrm{BALB} / \mathrm{c}$ nocaute para o receptor C5a, a infecção por L. major. Nesse modelo de estratégia de evasão, L. major levou à ativação do sistema Complemento por suprimir a imunidade mediada por célula induzida pelo TLR. 
Um dos importantes aspectos de doenças como a leishmaniose é a imunidade concomitante, resultante do longo tempo de persistência do patógeno no hospedeiro. A persistência de L. major após cura, em pele de camundongos resistentes $\mathrm{C} 57 \mathrm{BL} / 6$, é controlada por uma população de células $\mathrm{T}$ regulatórias $\mathrm{CD} 4{ }^{+} \mathrm{CD} 25^{+}$. Durante infecção por $L$. major, essas células acumulam-se na derme, onde suprimem, por mecanismos dependentes ou não de IL-10, a habilidade de células $\mathrm{T}$ efetoras $\mathrm{CD} 4^{+} \mathrm{CD} 25^{-}$eliminarem o parasito no sítio de infecção. A imunidade obtida em camundongo com atividade prejudicada de IL-10 é seguida da perda de imunidade à reinfecção, indicando que o equilíbrio estabelecido entre células $\mathrm{T}$ efetoras e reguladoras nos sítios de infecção crônica podem refletir estratégias de sobrevivência do parasito e do hospedeiro (Belkaid et al., 2002). A compreensão dessa complexa interação entre hospedeiro e parasito poderá influenciar no desenvolvimento de novas estratégias de profilaxia e terapia.

\section{ABSTRACT}

Immunological mechanisms in the cellular and humoral response in the American tegumentary leishmaniasis

The American tegumentary leishmaniasis is an anthropozoonotic disease caused by different Leishmania species, whose clinical manifestations varies from benign selfhealing cutaneous lesions to severe mutilating mucocutaneous lesions. Leishmania infections may lead to a specific immunological response by the host, characterized by the increase of $\mathrm{CD}^{+}$cells, showing a profile of cytokine Th1 or Th2. Infections by $L$. major, the best model studied, in resistant mice (C57BL/6, CBA, C3H) are associated with a Th1 response, whereas in susceptible mice (BALB/c), with a Th2 type response. Nevertheless the immune response in humans is not as well characterized as the response in mice and it involves the role of cytokines, costimulatory molecules and sand flies saliva. Altogether with the existence of an immunological response by the host, the survival and the persistence of the parasites depend of escaping strategies from adaptive immune response.

KEYWORDS: American tegumentary leishmaniasis. Leishmania. Cytokines. Immune response.

\section{REFERÊNCIAS}

1. Aebischer T. Recurrent cutaneous leishmaniasis: a role for persistent parasites? Parasitol Today 10: 25-28, 1994.

2. Amato VS, Andrade Jr HF, Duarte MIS. Mucosal leishmaniasis: in situ characterization of the host inflammatory response, before and after treatment. Acta Trop 85: 39-49, 2003.

3. Antonelli LR, Dutra WO, Almeida RP, Bacellar O, Gollob KJ. Antigen specific correlations of cellular immune responses in human leishmaniasis suggests mechanisms for immunoregulation. Clin Exp Immunol 136: 341-348, 2004. 
4. Awasthi A, Mathur, RK, Saha B. Immune response to Leishmania infection. Indian J Med Res 119: 238-258, 2004.

5. Bacellar O, Lessa H, Schriefer A, Machado P, Ribeiro de Jesus A, Dutra WO, Gollog KJ, Carvalho EM. Up-regulation of Th1-type responses in mucosal leishmaniasis patients. Infect Immun 70: 6734-6740, 2002.

6. Becker I, Salaiza N, Aguirre M, Delgado J, Carrillo-Carrasco N, Kobeh LG, Ruiz A, Cervantes R, Torres AP, Cabrera N, González A, Maldonado C, Isibasi A. Leishmania lipophosphoglycan (LPG) activates NK cells through toll-like receptor-2. Mol Biochem Parasitol 130: 65-74, 2003.

7. Belkaid Y, Piccirillo CA, Mendez S, Shevach E, Sacks DL. CD4 $4^{+} D 25^{+}$regulatory T cells control Leishmania major persistence and immunity. Nature 420: 502-507, 2002.

8. Bogdan C, Gessner A, Solbach W, Rollinghoff M. Invasion, control and persistence of Leishmania parasites. Curr Opin Immunol 8: 517-525, 1996.

9. Bogdan C, Rollinghoff M. The immune response to Leishmania: mechanisms of parasite control and evasion. Int J Parasitol 28: 121-134, 1998.

10. Bomfim G, Nascimento C, Costa J, Carvalho EM, Barral-Netto M, Barral A. Variation of cytokine patterns related to therapeutic responses in diffuse cutaneous leishmaniasis. Exp Parasitol 84: 188-194, 1996.

11. Borges MM, Campos-Neto A, Sleath P, Grabstein KH, Morrissey PJ, Skeiky AW, Reed SG. Potent stimulation of the innate immune system by a Leishmania braziliensis recombinant protein. Infect Immun 69: 5270-5277, 2001.

12. Bourreau E, Prévot G, Gardon J, Pradinaud R, Launois P. High intralesional interleukin-10 messenger RNA expression in localized cutaneous leishmaniasis is associated with unresponsiveness to treatment. J Infect Dis 184: 1628-1630, 2001.

13. Brodskyn CI, Dekrey GK, Titus RG. Influence of costimulatory molecules $\mathrm{n}$ immune response to Leishmania major by human cells in vitro. Infect Immun 69: 665-672, 2001.

14. Cáceres-Dittmar G, Tapia FJ, Sãnchez MA, Yamamura M, Uyemura K, Modlin RL, Bloom BR, Convit J. Determination of the cytokine profile in American cutaneous leishmaniasis using the polymerase chain reaction. Clin Exp Immunol 91: 500-505, 1993.

15. Carrera L, Gazzinelli RT, Badolato R, Hieny S, Muller W, Kuhn R, Sacks DL. Leishmania promatigotes selectively inhibit interleukin 12 induction in bone marrow-derived macrophages from susceptible and resistant mice. J Exp Med 183: 515-526. 1996.

16. Carvalho EM, Barral A, Costa JM, Bittencourt A, Marsden P. Clinical and disseminated immunopathological aspects of disseminated cutaneous leismaniasis. Acta Trop 56: 315-325, 1994.

17. Coler RN \& Reed SG. Second-generation vaccines against leishmaniasis. Trends Parasitol 21: 244249,2005 .

18. Cunningham AC. Parasitic adaptive mechanisms in infection by Leishmania. Exp Mol Pathol 72: 132-141, 2002.

19. da-Cruz AM, Bittar R, Mattos M, Oliveira-Neto MP, Nogueira R, Pinho-Ribeiro V, Azeredo-Coutinho RB, Coutinho SG. T-Cell-Mediated immune responses in patients with cutaneous or mucosal leishmaniasis: Long-term evaluation after therapy. Clin Diagn Lab Immunol 9: 251-256, 2002.

20. de Veer MJ, Curtis JM, Baldwin TM, DiDonato JA, Sexton A, McConville MJ, Handman E, Schofield L. MyD88 is essential for clearance of Leishmania major: possible role for lipophosphoglycan and toll-like receptor 2 signaling. Eur J Immunol 33: 2822-2831, 2003.

21. Ding L, Linsley PS, Huang LY, Germain RN, Shevach EM. IL-10 inhibits macrophage costimulatory activity by selectively inhibiting the up-regulation of B7 expression. J Immunol 151: 1224-1234, 1993.

22. Elloso MM, Scott P. Expression and contribution of B7.1 (CD80) and B7.2 (CD86) in the early immune response to Leishmania major infection. J Immunol 162: 6708-6715, 1999.

23. Forget $\mathrm{G}$. Étude des mécanismes de régulation négative utilisés par Leishmania pour contrer la réponse immunitaire innée. Faculte de Médecine, 2004. Disponível em: www.theses.ulaval. ca/2004/21419/21419.html. Acessado em: outubro/04.

24. Fundação Nacional de Saúde-Funasa, Ministério da Saúde-MS. Guia de Vigilância Epidemiológica. Vol.2, 5a Ed., p.399, 2002. 
25. Garcia AL, Kindt A, Quispe-Tintaya, KW, Bermudez, H, Llanos A, Arevalo J, Bañuls AL, De Doncker S, Le Ray D, Dujardin JC. American tegumentary leishmaniasis: antigen-gene polymorphism, taxonomy and clinical pleomorphism. Infect Genet Evol 5: 109-116, 2005.

26. Gillespie RD, Mbow ML, Titus RG. The immunomodulatory factors of bloodfeeding arthropod saliva. Parasite Immunol 22: 319-331, 2000.

27. Gollob KJ, Antonelli LRV \& Dutra WO. Insights into CD4+ memory T cells following Leishmania infection. Trends Parasitol 21: 347-350, 2005.

28. Gramiccia M \& Gradoni L. The current status of zoonotic leishmaniases and approaches to disease control. Int J Parasitol 35: 1169-1180, 2005.

29. Grimaldi Jr G, Tesh RB. Leishmaniasis of the New World: current concepts and implications for future research. ClinMicrobiol Rev 6: 230-250, 1993.

30. Handman E, Ceredig R, Mitchell GF. Murine cutaneous leishmaniasis: disease patterns in intact and nude mice of various genotypes and examination of some differences between normal and infected macrophages. Aust J Exp Biol Med Sci 75: 9-29, 1979.

31. Hawlisch H, Belkaid Y, Baelder R, Hildeman D, Gerard C, Köhl J. C5a negatively regulates toll-like receptor 4-induced immune responses. Immunity 22: 415-426, 2005.

32. Heinzel FP, Sadick MD, Holaday BJ, Coffman RL, Locksley RM. Reciprocal expression of IFN- $\gamma$ or IL-4 during the resolution or progression of murine leishmaniasis. Evidence for expression of distinct helper T cell subsets. J Exp Med 169: 59-72, 1989.

33. Himmelrich H, Launois P, Maillard I, Biedermann T, Tacchini-Cottier F, Locksley RM, Röcken M, Louis JA. In BALB/c mice, IL-4 production during the initial phase of infection with Leishmania major is necessary and sufficient to instruct Th2 cell development resulting in progressive disease. J Immunol 164: 4819-4825, 2000.

34. Hoare CA, Wallace FC. Developmental stages of trypanosomatid flagellates: A new terminology. Nature 212: 1358-1996, 1966.

35. Howard JG, Hale C, Chan-Liew WL. Immunological regulation of experimental cutaneous leishmaniasis. Immunogenetic aspects of a susceptibility to Leishmania tropica in mice. Parasite Immunol 2: 303-314, 1980.

36. Junqueira Pedras M, Orsini M, Castro M, Passos VMA, Rabello A. Antibody subclass profile against Leishmania braziliensis and Leishmania amazonensis in the diagnosis and follow-up of mucosal leishmaniasis. Diagn Microbiol Infect Dis 47: 477-485, 2003.

37. Kamhawi S. The biological and immunomodulatory properties of sand fly saliva and its role in the establishment of Leishmania infections. Microbes Infect 2: 1765-1773, 2000.

38. Kane MM \& Mosser DM. The role of IL-10 in promoting disease progression in leishmaniasis. J Immunol 166: 1141-1147, 2001.

39. Kar K. Serodiagnosis of leishmaniasis. Crit Rev Microbiol 21: 123-152, 1995.

40. Kemp M. Regulator and effector functions of T-cell subsets in human Leishmania infections. APMIS Suppl 68:1-33, 1997.

41. Khamesipour A, Dowlati Y, Asilian A, Hashemi-Fesharki R, Javadi A, Noazin S, Modabber F. Leishmanization: use of an old method for evaluation of candidate vaccines against leishmaniasis. Vaccine 23: 3642-3648, 2005.

42. Kima PE, Constant SL, Hannum L, Colmenares M, Lee KS, Haberman AM, Shlomchik MJ, McMahon-Pratt D. Internalization of Leishmania mexicana complex amastigotes via the $\mathrm{Fc}$ receptor is required to sustain infection in murine cutaneous leishmaniasis. $J$ Exp Med 191: 1063-1067, 2000.

43. Korbel DS, Finney OC, Riley EM. Natural killer cells and innate immunity to protozoan pathogens. Int J Parasitol 34: 1517-1528, 2004.

44. Lainson R \& Shaw JJ. Evolution, classification and geographical distribution. In: Peters WJ, KillickKendrick (Eds). The leishmaniasis in Biology and Medicine. Academic Press, London. 1-120, 1987.

45. Laskay T, Röllinghoff M, Solbach W. Natural killer cells participate in the early defense against Leishmania major infectionin mice. Eur J Immunol 23: 2237-2241, 1993.

46. Liew FY. Regulation of cell-mediated immunity in Leishmaniasis. Curr Top Microbiol Immunol 155: 53-64, 1990. 
47. Locksley RM, Heinzel FP, Sadick MD, Holaday BS, Gardner KD Jr. Murine cutaneous leishmaniasis: susceptibility correlates with differential expansion of helper T-cell subsets. Ann Inst Immunol 138: 744-749, 1987.

48. Louzir H, Melby PC, Salah AB, Marrakchi H, Aoun K, Ismail RB, Dellagi K. Immunologic determinants of disease evolution in localized cutaneous leishmaniasis due to Leishmania major. $J$ Infect Dis 177: 1687-1695, 1998.

49. Maasho K, Sanchez F, Schurr E, Hailu A, Akuffo H. Indications of the protective role of natural killer cells in human cutaneous leishmaniasis in an area of endemicity. Infect Immun 66: 2698-2704, 1998.

50. Machado-Pinto J, Pinto J, Costa CA, Genaro O, Marques MJ, Modabber F, Mayrink W. Immunochemotherapy for cutaneous leishmaniasis: a controlled trial using killed Leishmania (Leishmania) amazonensis vaccine plus antimonial. Int J Dermatol 41: 73-78, 2002.

51. Manson-Bahr PE. Diagnosis. In: The leishmaniasis. London, Peters W. \& Killick-Kendrick R, 2: 703-728, 1987.

52. Marsden PD. Mucosal leishmaniasis ("espundia" Escornel, 1911) Trans R Soc Trop Med Hyg 80: 859-876, 1986.

53. Marzochi MCA. Curso-Doenças Infecto-Parasitárias. Leishmanioses no Brasil: as leishmanioses tegumentares. J Bras Med 63: 82-104, 1992.

54. Matos DS, Azeredo-Coutinho RBG, Schubach A, Conceição-Silva F, Baptista C, Moreira JS, Mendonça SCF. Differential interferon- $\gamma$ production characterizes the citokyne responses to Leishmania and Mycobacterium leprae antigens in concomitant mucocutaneous leishmaniasis and lepromatous leprosy. Clin Infect Dis 40: 5-12, 2005.

55. Mayrink W, da Costa CA, Magalhães PA, Melo MN, Dias M, Lima AO, Michalick MS, Williams P. A field trial of a vaccine against American dermal leishmaniasis. Trans $R$ Soc Trop Med Hyg 73: 385-387, 1979.

56. Mayrink W, Magalhães PA, Michalick MSM, da Costa CA, Lima AO, Melo MN, Toledo VP, Nascimento E, Dias M, Genaro O, et al. Immunotherapy as a treatment of American cutaneous leishmaniasis: preliminary studies in Brazil. Parassitologia (Rome) 34: 159-165, 1992.

57. Mayrink W, Botelho ACC, Magalhães PA, Batista SM, Lima AO, Genaro O, da Costa CA, Melo MN, Michalick MSM, Williams P, Dias M, Caiaffa WT, Nascimento E, Machado-Coelho GLL. Immunotherapy, immunochemotherapy and chemotherapy for American cutaneous leishmaniasis treatment. Rev Soc Bras Med Trop 39: 14-21, 2006.

58. Mbow ML, Bleyenberg JA, Hall LR, Titus RG. Phlebotomus papatasi sand fly salivary gland lysate down-regulates a Th1, but up-regulates a Th2, response in mice infected with Leishmania major. J Immunol 161: 5571-5577, 1998.

59. Mendonça MG, Brito MEF, Rodrigues EH, Bandeira V, Jardim ML, Abath FG. Persistence of Leishmania parasites in scars after clinical cure of American cutaneous leishmaniasis: is there a sterile cure? J Infect Dis 189: 1018-1023, 2004.

60. Netea MG, Van der Meer JWM, Kullberg BJ. Toll-like receptors as an escape mechanism from the host defense. Trends Microbiol 12: 484-488, 2004.

61. Nylen S, Maasho K, Söderstrom K, Ilg T, Akuffo H. Live Leishmania promastigotes can directly activate primary human natural killer cells to produce interferon-gama. Clin Exp Immunol 131: 457, 2003.

62. O'Neil CE, Labrada M, Saravia NG. Leishmania (Viannia) panamensis - specific IgE and IgA antibodies relation to expression of human tegumentary leishmaniasis. Am J Trop Med Hyg 49: 181-188, 1993.

63. Pirmez C, Yamamura M, Uyemura K, Paes-Oliveira M, Conceição-Silva F, Modlin RL. Cytokine patterns in the pathogenesis of human leishmaniasis. J Clin Invest 91: 1390-1395, 1993.

64. Pleass RJ \& Woof JM. Fc receptors and immunity to parasites. Trends Parasitol 17: 545-551, 2001.

65. Ribeiro-de-Jesus A, Almeida RP, Lessa H, Bacellar O, Carvalho EM. Cytokine profile and pathology in human leishmaniasis. Braz J Med Biol Res 31: 143-148, 1998.

66. Rodriguez V, Centeno M \& Ulrich M. The IgG isotypes of specific antibodies in patients with American cutaneous leishmaniasis; relationship to the cell-mediated immune response. Parasite Immunol 18: 341-345, 1996. 
67. Rogers KA, Dekrey GK, Mbow ML, Gillespie RD, Brodskyn CI, Titus RG. Type 1 and type 2 responses to Leishmania major. FEMS Microbiol Lett 209: 1-7, 2002.

68. Sacks D, Sher A. Evasion of innate immunity by parasitic protozoa. Nature Immunol 3: 1041-1047, 2002.

69. Salaiza-Suazo N, Volkow P, Tamayo RP, Moll H, Gillitzer R, Pérez-Torres A, Pérez-Montfort R, Domínguez JD, Velasco-Castrejón O, Crippa M, Becker I. Treatment of two patients with diffuse cutaneous leishmaniasis caused by Leishmania mexicana modifies the immunohistological profile but not the disease outcome. Trop Med Int Health 4: 801-811, 1999.

70. Scharton TM, Scott P. Natural killer cells are a source of interferon- $\gamma$ that drives differentiation of CD4+ T cell subsets and induces early resistance to Leishmania major in mice. J Exp Med 178: 567-577, 1993.

71. Scott P, Farrel JP. Experimental Cutaneous Leishmaniasis: Induction and Regulation of T Cells following Infection of Mice with Leishmania major. Chem Immunol 70: 60-80, 1998.

72. Sousa-Atta MLB, Salame GS, D'Oliveira Jr A, Almeida RP, Atta AM, Carvalho EM. Immunoglobulin E antileishmanial antibody response in cutaneous leishmaniasis. Clin Diagn Lab Immunol 9: 101-104, 2002.

73. Souza MA, Silva AG, Afonso-Cardoso SR, Favoreto Junior S, Ferreira MS. Perfil de isotipos de imunoglobulinas e subclasses de IgG na leishmaniose tegumentar americana. Rev Soc Bras Med Trop 38: 137-141, 2005.

74. Trujillo C, Ramirez R, Vélez ID, Berberich C. The humoral immune response to the kinetoplastid membrane protein-11 in patients with American leishmaniasis and chagas disease: prevalence og IgG subclasses and mapping of epitopes. Immunol Lett 70: 203-209, 1999.

75. Vieira MG, Oliveira F, Arruda S, Bittencourt AL, Barnosa AAJr, Barral-Netto M, Barral A. B-cell infiltration and frequency of cytokine producing cells differ between localized and disseminated human cutaneous leishmaniases. Mem Inst Oswaldo Cruz 97: 979-83, 2002.

76. Wang ZE, Reiner SL, Zheng S, Dalton DK, Locksley RM. CD4 effector cells default to the Th2 pathway in interferon- $\gamma$-deficient mice infected with Leishmania major.J Exp Med 179: 1367-1371, 1994.

77. World Health Organization (WHO). Geographical distribution. 2003. Disponível em: $<$ www.who.int/emc/diseases/leish/leishgeo1.html> . Acessado em: Maio 2004.

78. Zambrano-Villa S, Rosales-Borjas D, Carrero JC, Ortiz-Ortiz L. How protozoan parasites evade the immune response. Trends Parasitol 18: 272-278, 2002.

79. Zaph C, Uzonna J, Beverley SM, Scott P. Central memory T cells mediate long-term immunity to Leishmania major in the absence of persistent parasites. Nat Med 10: 1104-1110, 2004. 
PRÓXIMOS EVENTOS NA ÁREA DE PATOLOGIA TROPICAL E SAÚDE PÚBLICA

XVI International AIDS Conference (AIDS 2006). Toronto, Canadá, 13 a 18 de agosto de 2006. Informações: info@aids2006.org

$11^{\circ}$ Congresso Mundial de Saúde Pública e $8^{\circ}$ Congresso Brasileiro de Saúde Coletiva. Rio de Janeiro, RJ, 21 a 25 de agosto de 2006. Informações: www.wfphacongress06.com

IX Simpósio Internacional sobre Control Epidemiológico de Vectores. Buenos Aires, Argentina, 29 a 30 de agosto de 2006. Informações: www.mundosano.org

$14^{\circ}$. Congresso Brasileiro de Parasitologia Veterinária e $2^{\circ}$ Simpósio LatinoAmericano de Rickettsioses. Ribeirão Preto, SP, 3 a 6 de setembro de 2006. Informações: www.cbpv.com.br/congresso

Conferência Brasileira de HIV/AIDS e Hepatites Virais. Fortaleza, CE, 10 a 13 de setembro de 2006. Informações: www.cbah2006.com.br

X Congresso Brasileiro de Controle de Infecção e Epidemiologia Hospitalar. Porto Alegre, RS, 11 a 15 de setembro de 2006. Informações: E-mail: inscriçoes@abev.com.br

VI Congresso da SBDST e II Congresso Brasileiro de AIDS. Santos, SP, 17 a 20 de setembro de 2006. Informações: www.dstsaopaulo.org.br

XXII Reunião Anual de Pesquisa Aplicada em Doença de Chagas e Leishmanioses. Uberaba, MG, de 27 a 29 de outubro de 2006. Informações: E-mail: reuniaouberaba@mednet.com.brou dalmo@mednet.com.br

XLIII Congresso da Sociedade Brasileira de Medicina Tropical, Campos do Jordão, SP, 11 a 15 de março de 2007. Informações: www.sbmt.org.br ou atendimento@perfectaeventos.com.br 